\title{
Corrigendum to "Effects of Shenfu Injection in the Treatment of Septic Shock Patients: A Multicenter, Controlled, Randomized, Open-Label Trial"
}

\author{
Yi Li, ${ }^{1}$ Xinchao Zhang, ${ }^{2}$ Peihong Lin, ${ }^{3}$ Haibo Qiu, ${ }^{4}$ Jie Wei, ${ }^{5}$ Yu Cao, ${ }^{6}$ Shuming Pan, \\ Joseph Walline, ${ }^{8}$ Chuanyun Qian, ${ }^{9}$ Zhigang Shan, ${ }^{10}$ and XueZhong $\mathbf{Y u}^{1}$ \\ ${ }^{1}$ Emergency Department, Peking Union Medical College Hospital, Beijing 100730, China \\ ${ }^{2}$ Emergency Department, Beijing Hospital, National Center of Geriatrics, Beijing 100730, China \\ ${ }^{3}$ Emergency Department, The First Affiliated Hospital of Fujian Medical University, Fuzhou 350005, China \\ ${ }^{4}$ Department of Critical Care Medicine, Zhongda Hospital, School of Medicine, Southeast University, Nanjing 210009, China \\ ${ }^{5}$ Emergency Department, Renmin Hospital of Wuhan University, Wuhan 430060, China \\ ${ }^{6}$ Emergency Department, West China Hospital of Sichuan University, Chengdu 610041, China \\ ${ }^{7}$ Emergency Department, Xinhua Hospital, Shanghai Jiao Tong University School of Medicine, Shanghai 200092, China \\ ${ }^{8}$ Emergency Department, Saint Louis University, Saint Louis, MO 63110, USA \\ ${ }^{9}$ Emergency Department, The First Affiliated Hospital of Kunming Medical University, Kunming 650032, China \\ ${ }^{10}$ Emergency Department, PLA 263 Hospital, Beijing 101149, China
}

Correspondence should be addressed to XueZhong Yu; erxuezhongyu@sina.cn

Received 16 August 2016; Accepted 5 September 2016

Copyright ( $) 2016 \mathrm{Yi} \mathrm{Li}$ et al. This is an open access article distributed under the Creative Commons Attribution License, which permits unrestricted use, distribution, and reproduction in any medium, provided the original work is properly cited.

In the article titled "Effects of Shenfu Injection in the Treatment of Septic Shock Patients: A Multicenter, Controlled, Randomized, Open-Label Trial” [1], affiliations 2, 4, 5, 6, 7, 8,9 , and 10 were incorrect. The revised affiliations are shown above.

\section{References}

[1] Y. Li, X. Zhang, P. Lin et al., "Effects of Shenfu injection in the treatment of septic shock patients: a multicenter, controlled, randomized, open-label trial," Evidence-Based Complementary and Alternative Medicine, vol. 2016, Article ID 2565169, 9 pages, 2016. 


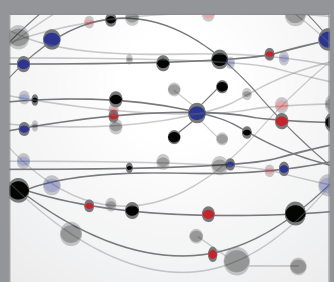

The Scientific World Journal
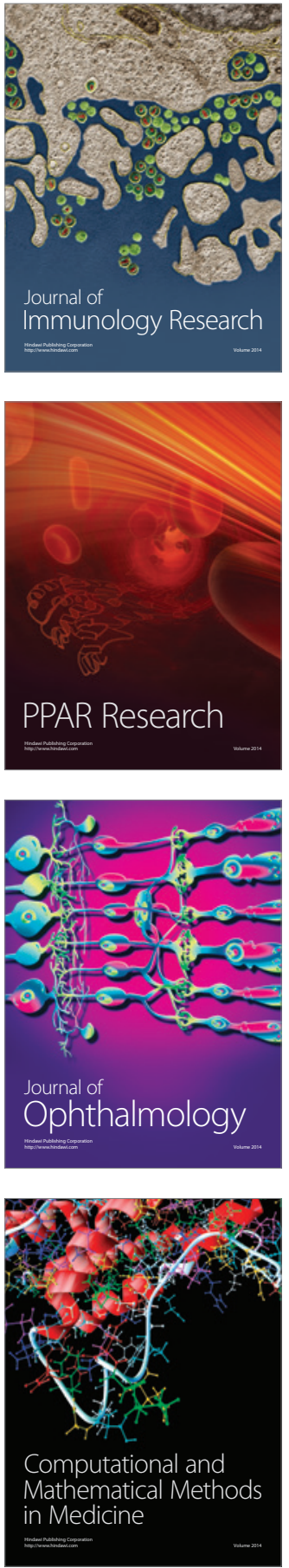

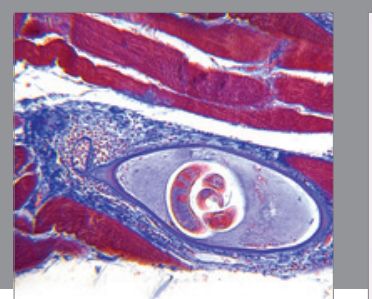

Gastroenterology Research and Practice

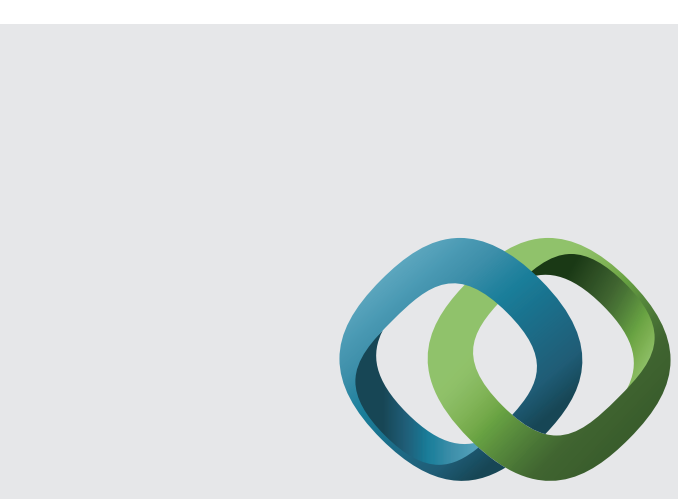

\section{Hindawi}

Submit your manuscripts at

http://www.hindawi.com
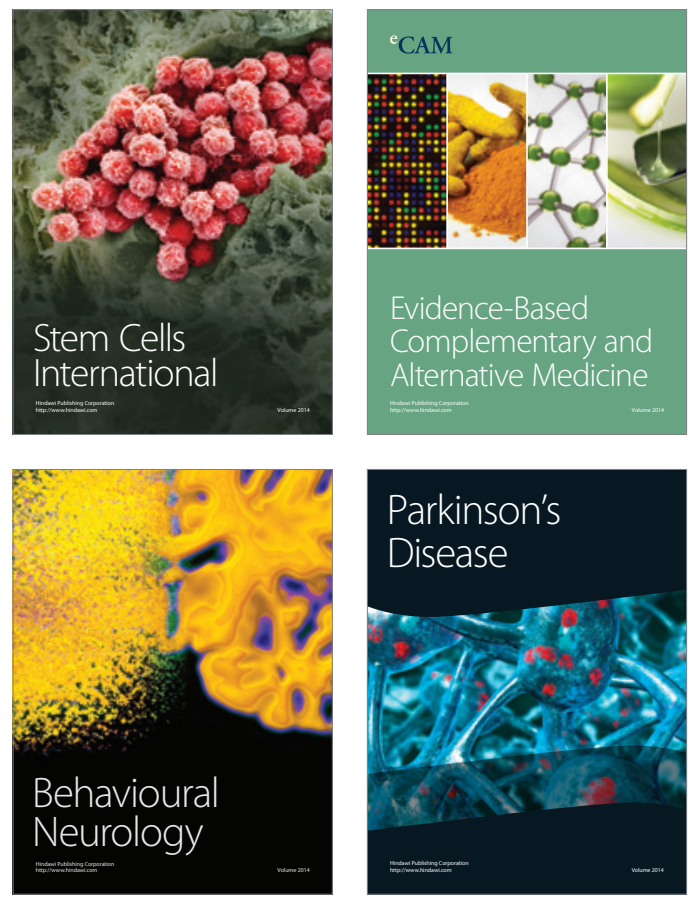
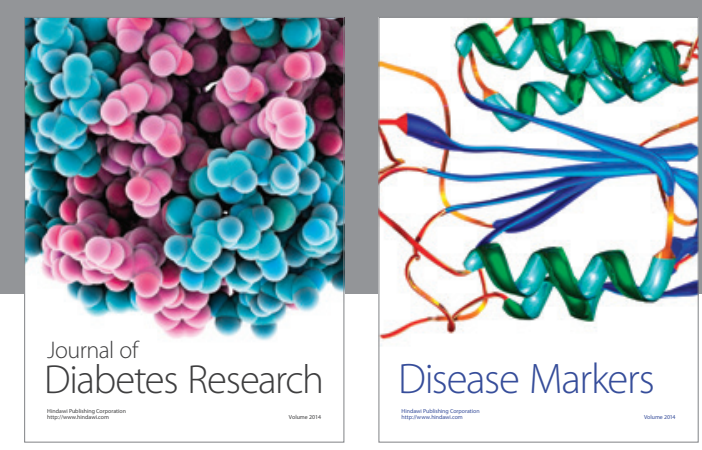

Disease Markers
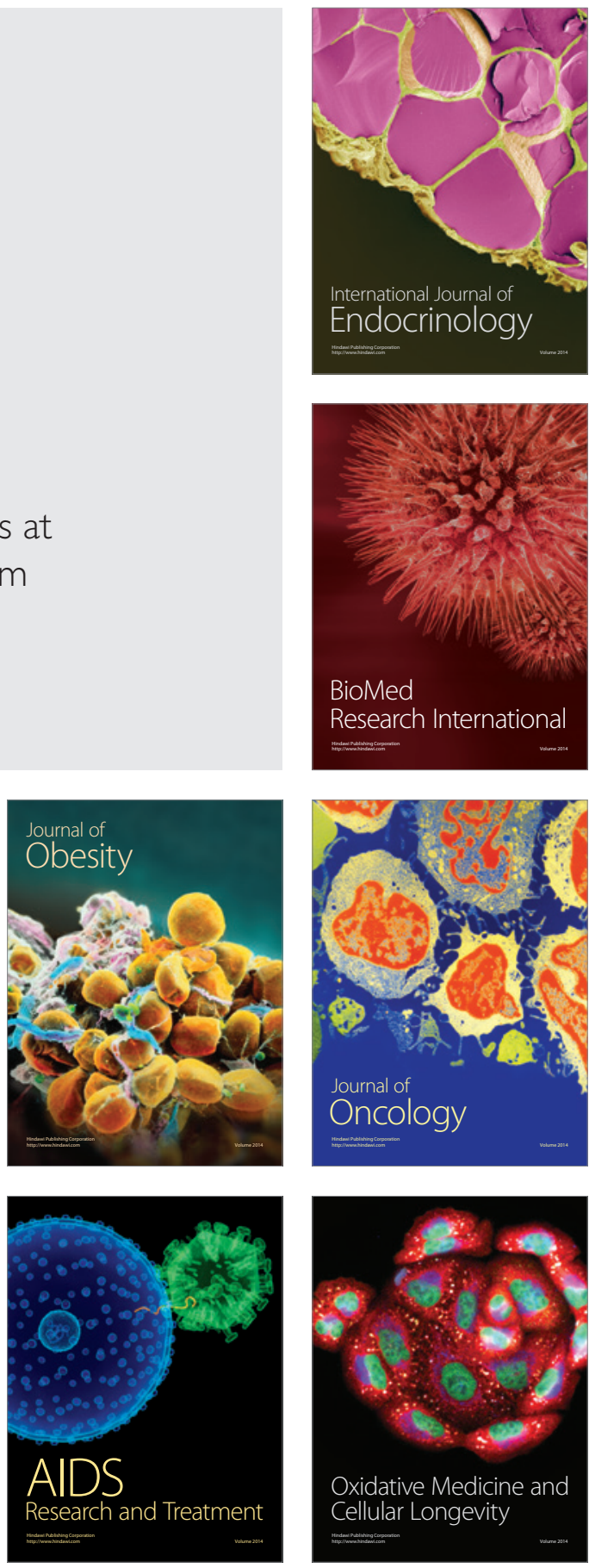